\title{
Comparison of numerical image reconstruction methods in holography
}

\author{
Zoltán Garaguly*‡, Miklós Kozlovszky ${ }^{\dagger}$, and Levente Kovács ${ }^{\ddagger}$ \\ ${ }^{*}$ Doctoral School of Applied Informatics and Applied Mathematics, Obuda University, Budapest, Hungary \\ ${ }^{\dagger}$ BioTech Research Center, University Innovation and Research Center, Obuda University, Hungary

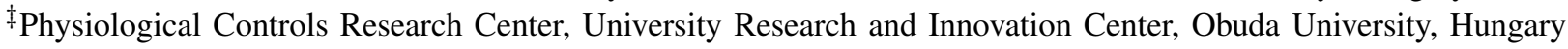

\begin{abstract}
In favour of the reconstruction of the real or virtual image's phase and intensity, the complete digital holographic images are being processed. This reconstruction takes place with the numerical definition of the diffraction integral. One of the possible realization is the Fresnel approximation, which employs a sole Fourier-transformation. Another method is to interpret the diffraction formula as a convolution integral, and if we calculate the formula, it will be doubled or tripled because of the transformation. The impulse response of wave fields should be represented in this convolution approach, from which the Fourier transform can be immediately determined. The impulse response as well as the Fourier transform can be immediately specified, or well approximated. The essential distinction between the Fresnel and convolution approach is the different size of resultant images. Furthermore, this size in case of the Fresnel process depends on the distance of the object and the sensor, as well as the wavelength of the illuminating light; but in the other case, it is invalid.
\end{abstract}

\section{INTRODUCTION}

Holography is a good way for recording and reconstructing optical wave fields. Holographic interferometry in metrology seems the most significant utilisation of holography, where, before and after variation, wave fields are correlated. Deformation fields and refraction index alterations are coded into distinct interference fringes [1]. The patterns of interference to the precision measurements are recorded with CCD or CMOS cameras and are evaluated numerically. At first the interference phase distribution is defined, from which, secondly, we can calculate the displacement of vector fields, deformations, stresses, refractive and physical quantities. The description of digital holographic reconstruction- based on diffraction theory- comes afterwards, after a short overview of the given requirements of holographic method and sampling principle. The numerical algorithm and the calculation of the diffraction integral is being implemented. The typical features and capabilities of calculations should be identified and compared. It is important, that not the numerically produced holograms were refined, but the optically produced holograms had been digitally recorded and after that these were reconstructed.

\section{THE FRESNEL APPROXIMATION}

On the following graph, the geometry for the description of Fresnel holography is presented:

We think that the plane surface on $(x, y)$ plane denote on $b(x, y)$ wave field. Let the hologram be on $(\xi, \eta)$ plane, $d$ distance from the surface of the object [3]. The reconstructed

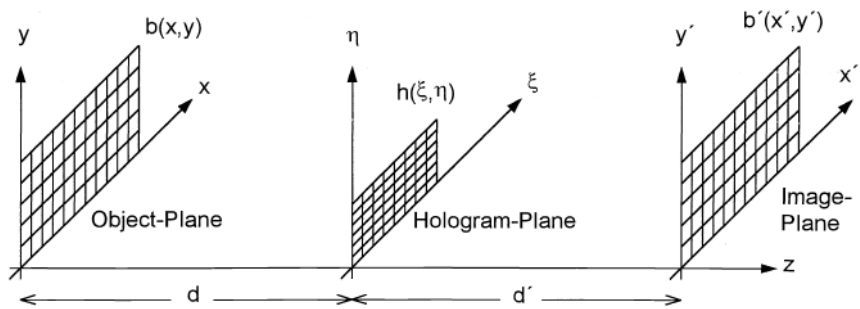

Fig. 1. Fresnel geometry [2].

real image is placed on $(x, y)$ plane which is distance $d$ from the plane of the hologram.

The interference in the hologram in each point is determined by angle $\theta$ between the reference wave and the object wave:

$$
\delta=\frac{\lambda}{2 \sin (\theta / 2)}
$$

where, $\delta$ the received ray distance and $\lambda$ denotes the used wave length. To the $\Delta \xi$ pixel distance sensor matrix the sampling rule requires at least 2 pixels per ray period as $2 \Delta \xi<\delta$ or

$$
\theta<\frac{\lambda}{2 \Delta \xi}
$$

since, for the small $\theta, \sin \theta \approx \tan \theta \approx \theta$. Small angles required by the sampling rule can be used at survey of small objects; remote objects from the sensor matrix or at magnification of the wave field with negative lens [7].

Parallel or divergent reference waves can be used in digital holography with normal or leaning incidence onto the sensor matrix. Largely, which is the most suitable for numerical evaluation, is the plane wave, generally with spatially constant amplitude and phase. It can be modelled with $r(\xi, \eta)=1+0 i$ on the basis of real vectors.

Nevertheless, the optical reconstruction was executed by the illumination of the advanced hologram with the reference wave. Therefore, the digital hologram should be multiplied with the reference wave field in the hologram's plane and the diffraction pattern in the image plane should be calculated. The real image is represented by the complex field, if $d=d$, or the virtual image, if $d=-d$ is taken.

The Rayleigh-Sommerfeld equation disposes the diffracted field in the image plane [8]. 


$$
b^{\prime}\left(x^{\prime}, y^{\prime}\right)=\frac{1}{i \lambda} \iint h(\xi, \eta) r(\xi, \eta) \frac{\exp \{i k p\}}{\rho} \cos \Theta d \xi d \eta
$$

$$
\rho=\sqrt{d^{2}+\left(\xi-x^{\prime}\right)^{2}+\left(\eta-y^{\prime}\right)^{2}}
$$

Here $h(\xi, \eta)$ is the recorded hologram, $r(\xi, \eta)$ is the reference wave field, $k=2 / \lambda$ is the number of the wave, and $\cos \theta$ means the leaning factor which can normally be disposed with $\cos \theta=1$, thanks to the small angles between the normal hologram and the rays from the hologram to the real image points.

Whereas in case of (3), $d^{\prime}$ can replace $\rho$ in the denominator, until $d^{\prime}$ is bigger than $\left(\xi-x^{\prime}\right)$ and $\left(\eta-y^{\prime}\right), \rho, \rho$ in the numerator determines the spatially quickly changing phase. The same replacement in the numerator would cause unacceptable mistakes. Binomial expansion $\left(\sqrt{1-a}=1+\frac{1}{2} a-\frac{1}{8} a^{2}+-\ldots\right)$ is applied in the Fresnel approximation for the square root (4) which applies to small $a$. Preserving the first two conditions, we get:

$$
\rho \approx d^{\prime}\left[1+\frac{1}{2}\left(\frac{\xi-x^{\prime}}{d^{\prime}}\right)^{2}+\frac{1}{2}\left(\frac{\eta-y^{\prime}}{d^{\prime}}\right)^{2}\right]
$$

Afterwards the diffraction integral is the following:

$$
\begin{array}{r}
b^{\prime}\left(x^{\prime}, y^{\prime}\right)=\frac{1}{i \lambda d^{\prime}} \iint h(\xi, \eta) r(\xi, \eta) \\
\exp \left\{i k d^{\prime}\left[1+\frac{1}{2}\left(\frac{\xi-x^{\prime}}{d^{\prime}}\right)^{2}+\frac{1}{2}\left(\frac{\eta-y^{\prime}}{d^{\prime}}\right)^{2}\right]\right\} d \xi d \eta= \\
=\frac{\exp \left\{i k d^{\prime}\right\}}{i \lambda d^{\prime}} \iint h(\xi, \eta) r(\xi, \eta) \\
\exp \left\{\frac{i k}{2 d^{\prime}}\left[\left(\xi-x^{\prime}\right)^{2}+\left(\eta-y^{\prime}\right)^{2}\right]\right\} d \xi d \eta= \\
=\frac{\exp \left\{i k d^{\prime}\right\} \exp \left\{i \pi d^{\prime} \lambda\left(\nu^{2}+\mu^{2}\right)\right\}}{i \lambda d^{\prime}} \\
\iint h(\xi, \eta) r(\xi, \eta) \exp \left\{\frac{i \pi}{d^{\prime} \lambda}\left(\xi^{2}+\eta^{2}\right)\right\} \\
\exp \{-2 i \pi(\xi \nu+\eta \mu)\} d \xi d \eta
\end{array}
$$

if $\nu=\frac{x^{\prime}}{d \lambda}$ and $\mu=\frac{y^{\prime}}{d \lambda}$.

The diffracted field is the hologram's Fourier transform, as it is shown in (6)'s last line, multiplying with the reference wave and the chirp function $\left\{\frac{i \pi}{d^{\prime} \lambda}\left(\xi^{2}+\eta^{2}\right)\right\}$ Then, the result of this Fourier transformation with phase factor and the spatially constant intensity factor $1 /\left(i \lambda d^{\prime}\right)$ should be multiplied.

We take the discrete finite form of (6), for numerical evaluation and we omit the spatially constant factors.

$$
\begin{array}{r}
b^{\prime}(n, m)=\exp \left\{\frac{i \pi d^{\prime} \lambda}{N^{2}}\left(\frac{n^{2}}{\Delta \xi^{2}}+\frac{m^{2}}{\Delta \eta^{2}}\right)\right\} \\
\sum_{k=0}^{N-1} \sum_{l=0}^{N-1} h(k \Delta \xi, l \Delta \eta) r(k \Delta \xi, l \Delta \eta) \\
\exp \left\{\frac{i \pi}{d^{\prime} \lambda}\left(k^{2} \Delta \xi^{2}+l^{2} \Delta \eta^{2}\right)\right\} \times \exp \left\{2 i \pi\left(\frac{k n}{N}+\frac{l m}{N}\right)\right\}
\end{array}
$$

Or in short:

$$
b^{\prime}=z \cdot \mathcal{F}^{-1}\{h \cdot r \cdot w\}
$$

In these equations $h(k \Delta \xi, l \Delta \eta)$ signifies the quantised and digitalised hologram, $r(k \Delta \xi, l \Delta \eta)$ is the reference wave, $w(k \Delta \xi, l \Delta \eta)=\exp \left\{\frac{i \pi}{d^{\prime} \lambda}\left(k^{2} \Delta \xi^{2}+l^{2} \Delta \eta^{2}\right)\right\}$ is the two dimension finite chirp function, as well as $z\left(n \Delta x^{\prime}, m \Delta y^{\prime}\right)=$ $\exp \left\{\frac{i \pi d^{\prime} \lambda}{N^{2}}\left(\frac{n^{2}}{\Delta \xi^{2}}+\frac{m^{2}}{\Delta \eta^{2}}\right)\right\}=\left\{-\frac{i \pi}{d^{\prime} \lambda}\left(n^{2} \Delta x^{\prime 2}+m^{2} \Delta y^{\prime 2}\right)\right\}$. The reconstructed image's pixel size:

$$
\Delta x^{\prime}=\frac{d^{\prime} \lambda}{N \Delta \xi} \quad \Delta y^{\prime}=\frac{d^{\prime} \lambda}{N \Delta \eta}
$$

where, $N$ is the pixel number of the sensor matrix in each direction.

In most application the phase factor $z\left(n \Delta x^{\prime}, m \Delta y^{\prime}\right)$ is negligible, unless the reconstructions intensities are interested, or phase differences count, for instance, in holographic interferometry [4] [6]. $z$ does not depend on the evaluated hologram thus it provides the same phase shift in each point in different object positions reconstruction and drops out the holographic interferometry's process of phase deduction.

On the Fig. 2., a holographic record can be seen, made from the USAF 1951 resolution testing slide preparation, where the object is placed $4.5 \mathrm{~mm}$ from the sensor matrix, the pixelsize is $\Delta \xi=6.8 \mu \mathrm{m}$ and the wavelength is $\lambda=0.470 \mu \mathrm{m}$. The reference wave is: $r(k \Delta \xi, l \Delta n)=1+0 i$. The numerically reconstructed wave field; the reconstructed image can be seen on the Fig. 3. The d.c. condition of the high intensity Fresnel transformation covers the reconstructed field's central area, which can be eliminated with the methods described in [9].

\section{THE DIFFRACTION INTEGRAL, AS CONVOLUTION (CONVOLUTION APPROACH)}

The Rayleigh-Sommerfeld diffraction equation (3) is a superposition integral for a $b^{\prime}\left(x^{\prime}, y^{\prime}\right)$ wave field's reconstruction.

$$
b^{\prime}\left(x^{\prime}, y^{\prime}\right)=\iint h(\xi, \eta) r(\xi, \eta) g\left(x^{\prime}, y^{\prime}, \xi, \eta\right) d \xi d \eta
$$

where, a $g\left(x^{\prime}, y^{\prime}, \xi, \eta\right)$ impulse response is finitely given as follows: 


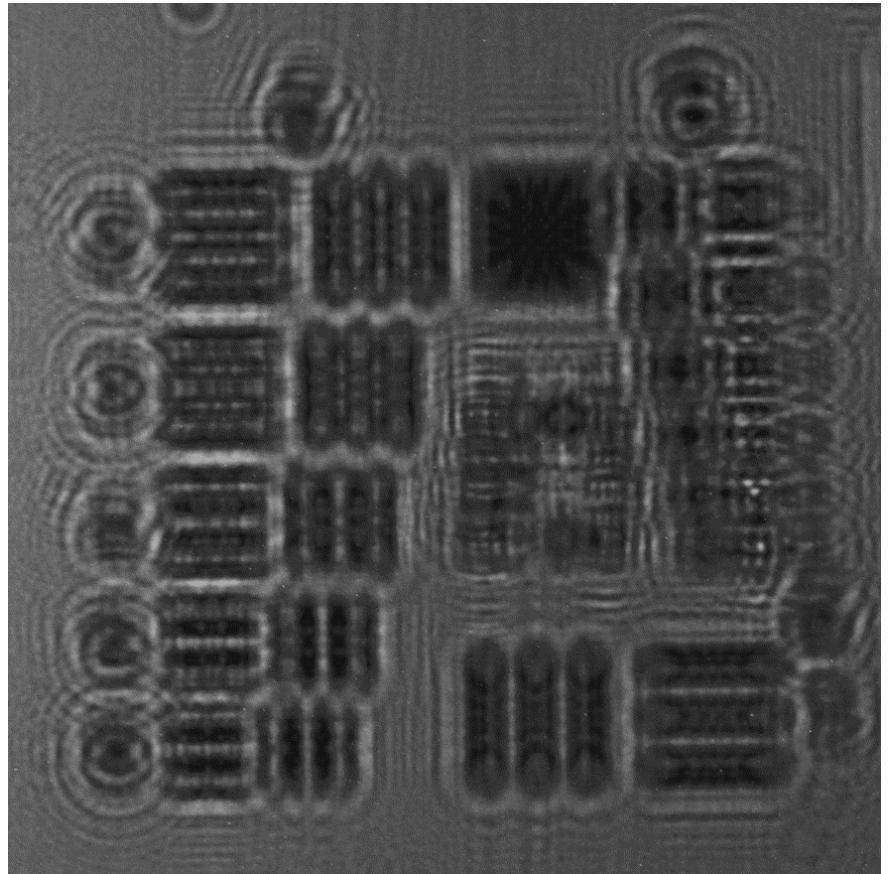

Fig. 2. USAF 1951 holographic image.

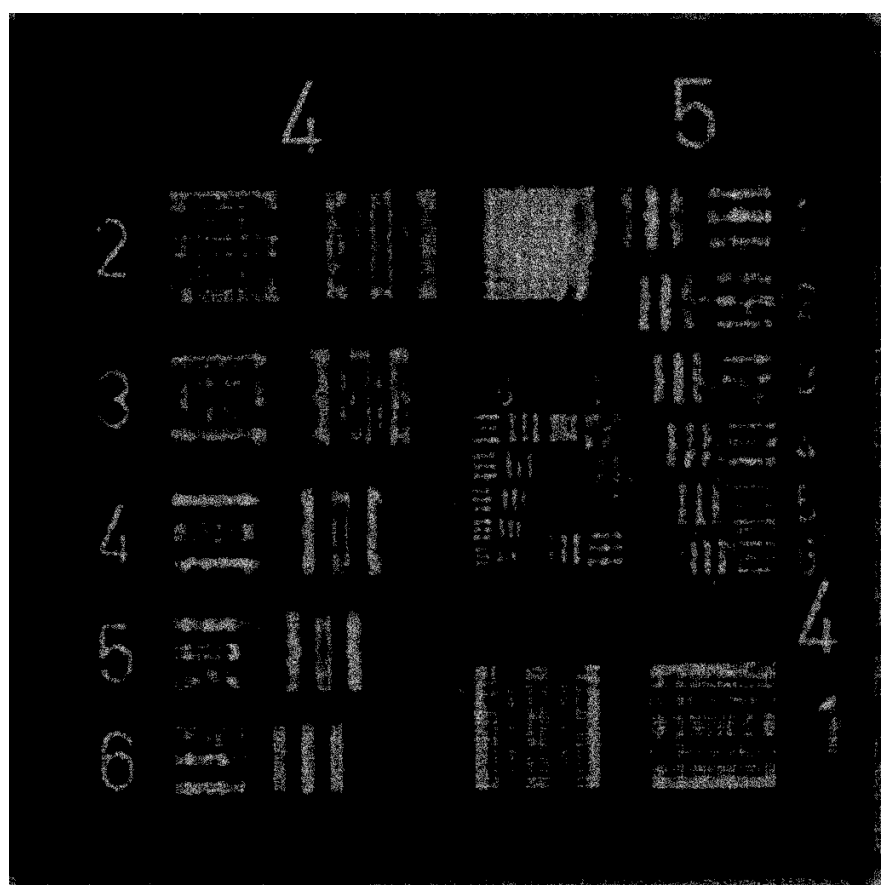

Fig. 3. USAF 1951 reconstructed image.

$$
\begin{array}{r}
g\left(x^{\prime}, y^{\prime}, \xi, \eta\right)=\frac{1}{i \lambda} \frac{\exp \{i k p\}}{\rho} \cos \Theta= \\
=\frac{d^{\prime}}{i \lambda} \frac{\exp \left\{i k \sqrt{d^{\prime 2}+\left(\xi-x^{\prime}\right)^{2}+\left(\eta-y^{\prime}\right)^{2}}\right\}}{d^{\prime 2}+\left(\xi-x^{\prime}\right)^{2}+\left(\eta-y^{\prime}\right)^{2}}
\end{array}
$$

Here $\cos \Theta=d^{\prime} / \rho$ has been applied. The second line of (11) shows that $\left.g\left(x^{\prime}, y^{\prime}, \xi, \eta\right)=g\left(x^{\prime}-\xi\right), y^{\prime}-\eta\right)$ describes the linear system which is spatially constant, in conclusion, the superposition integral equals to a convolution. It allows the economical calculation of the diffraction integral without estimation [5]. The convolution theorem claims that the convolution with the Fourier transformation of $h \cdot r, g$ is the result of $F\{h \cdot r\}$ and $F\{g\}$ individual transformations. Therefore $b^{\prime}\left(x^{\prime}, y^{\prime}\right)$ can be calculated with the first Fourier transformation of $h \cdot r$, which is then multiplied with the Fourier transformation of $g$, and then we take the result's inverse Fourier transform. All the three Fourier transformations are needed for the whole process, to which the FFT (Fast Fourier Transformation) algorithm is effectively applicable [5].

The impulse response's numerical realisation:

$g(k, l)=\frac{1}{i \lambda} \frac{\exp \left\{\frac{2 i \pi}{\lambda} \sqrt{d^{\prime 2}+(k-N / 2)^{2} \Delta \xi^{2}+(l-N / 2)^{2} \Delta \eta^{2}}\right\}}{\sqrt{d^{\prime 2}+(k-N / 2)^{2} \Delta \xi^{2}+(l-N / 2)^{2} \Delta \eta^{2}}}$

Because of symmetrical reasons, the $N / 2$ shift of coordinates takes place. Furthermore, the intensity condition $\cos \Theta=d^{\prime} / \rho$ is negligible, as $\cos \Theta$, based on $\Theta$, defined by (2) is less than $1 / 1000$, results the difference from 1 . (12) performs the free expansion of the impulse response function. The Fourier transform of $g(k, l)$ impulse response is the $G(n, m)$ transfer function. Thus, the free space propagation's transfer function can also be defined directly as follows:

$=\exp \left\{\frac{2 \pi i d^{\prime}}{\lambda} \sqrt{1-\frac{\lambda^{2}\left(n+\frac{N^{2} \Delta \xi^{2}}{2 d^{\prime} \lambda}\right)^{2}}{N^{2} \Delta \xi^{2}}-\frac{\lambda^{2}\left(m+\frac{N^{2} \Delta \eta^{2}}{2 d^{\prime} \lambda}\right)^{2}}{N^{2} \Delta \eta^{2}}}\right\}$

with this we have saved a Fourier transformation.

The survey of the first line of (6) shows that similarly the Fresnel transformation is a convolution, with the following impulse response:

$$
\begin{aligned}
& g_{F}\left(\xi-x^{\prime}, \eta-y^{\prime}\right)= \\
& =\frac{\exp \left\{i k d^{\prime}\right\}}{i \lambda d^{\prime}} \exp \left\{\frac{i \pi}{\lambda d^{\prime}}\left[\left(\xi-x^{\prime}\right)^{2}+\left(\eta-y^{\prime}\right)^{2}\right]\right\}
\end{aligned}
$$

The transfer function's Fresnel approximation:

$$
\begin{array}{r}
G_{F}(n, m)= \\
=\exp \left\{i \pi d ^ { \prime } \left[\frac{2}{\lambda}-\lambda\left(\frac{n}{N \Delta \xi}+\frac{N \Delta \xi}{2 d^{\prime} \lambda}\right)^{2}-\right.\right. \\
\left.\left.-\lambda\left(\frac{m}{N \Delta \eta}+\frac{N \Delta \eta}{2 d^{\prime} \lambda}\right)^{2}\right]\right\}
\end{array}
$$

The reconstructed image's pixel size, when we reconstruct with the product of the transfer function, is the following:

$$
\Delta x^{\prime}=\Delta \xi \quad \Delta y^{\prime}=\Delta \eta
$$


The emerged image covers image field $N \Delta \xi \times N \Delta \eta$ instead of a in comparison with the Fresnel transformation. Afterwards the bigger image size until $\left(d^{\prime}>\Delta \xi 2 / \lambda\right)$, demonstrates that there is even more data coded in the hologram, than it was at the reconstruction performed by the sole convolution integral. It seems impossible to extract this additional data, with setting distinct values for $(\xi, \eta)$ and $\left(x^{\prime}, y^{\prime}\right)$ plane. It would violate the field invariant, which is a necessary condition for the validity of the convolution theorem. Thus, the impulse response's application shifts the reconstructed field with $\left(s_{k} \Delta \xi, s_{l} \Delta \eta\right)$ vector [6].

$$
\begin{array}{r}
g\left(k+s_{k}, l+s_{l}\right)= \\
=\frac{1}{i \lambda} \\
\frac{\exp \left\{\frac{2 i \pi}{\lambda} \sqrt{d^{\prime 2}+\left(k-N / 2+s_{k}\right)^{2} \Delta \xi^{2}+\left(l-N / 2+s_{l}\right)^{2} \Delta \eta^{2}}\right\}}{\sqrt{d^{\prime 2}+\left(k-N / 2+s_{k}\right)^{2} \Delta \xi^{2}+\left(l-N / 2+s_{l}\right)^{2} \Delta \eta^{2}}}
\end{array}
$$

The extent of possible shifts is given by the sampling theorem, in compliance with (2).

On Fig. 4. the reconstruction of 4 separate shifts can be seen. The comparison on Fig. 3. clearly makes the different image sizes in contrast with the Fresnel-results visible.
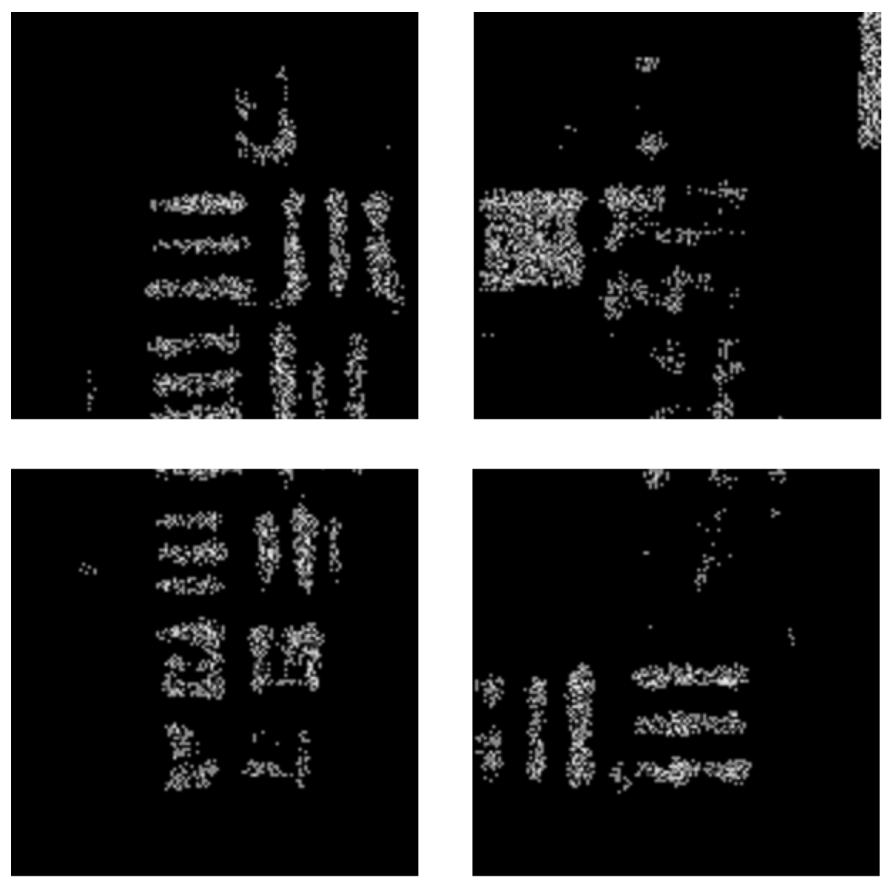

Fig. 4. Reconstructed images with the process of convolution and different shifts.

The image can be scaled as well, until the same scaling takes place on $(\xi, \eta)$ and $\left(x^{\prime}, y^{\prime}\right)$ planes. Black pixels can be added in order to increase the margin of $N \times N$ hologram and have a $2 N \times 2 N$ hologram. The $N \times N$ pixels in centre derives from the original hologram, whereas the surrounding pixels possess 0 intensity. The size of the reconstructed image is
$2 N \Delta \xi \times 2 N \Delta \eta$. There is an example on Fig. 5., where on the left side, the magnified hologram can be seen, as well as on the right side, the corresponding reconstructed image.
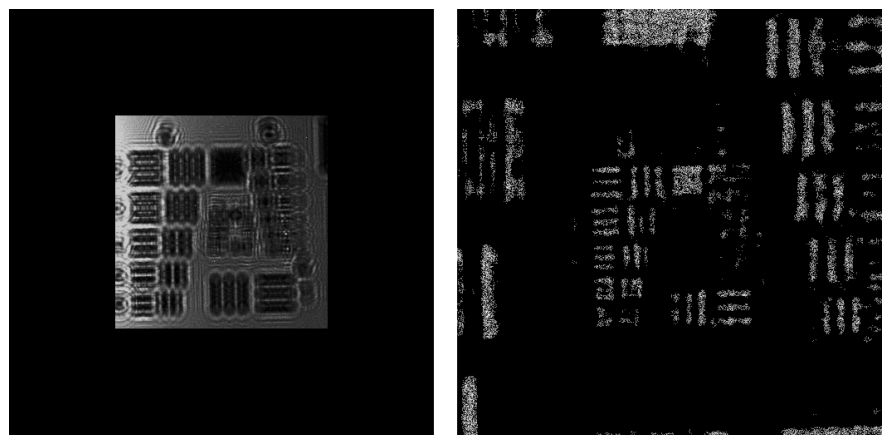

Fig. 5. Magnified hologram and the reconstructed image.

\section{COMPARISON OF THE RECONSTRUCTION METHODS}

Different realisations of the diffraction integral are shown in the following table:

TABLE I

METHODS

\begin{tabular}{|l|l|}
\hline Method & Algorithm \\
\hline $\begin{array}{l}\text { Fresnel approximation } \\
\text { (Chirp function) }\end{array}$ & $z \cdot \mathcal{F}^{-1}\{h \cdot r \cdot w\}$ \\
\hline $\begin{array}{l}\text { Fresnel approximation } \\
\text { (Impulse response) }\end{array}$ & $\mathcal{F}^{-1}\left\{\mathcal{F}\{h \cdot r\} \cdot \mathcal{F}\left\{g_{F}\right\}\right\}$ \\
\hline $\begin{array}{l}\text { Fresnel approximation } \\
\text { (Transfer function) }\end{array}$ & $\mathcal{F}^{-1}\left\{\mathcal{F}\{h \cdot r\} \cdot G_{F}\right\}$ \\
\hline $\begin{array}{l}\text { Diffraction integral } \\
\text { (Impulse response) }\end{array}$ & $\mathcal{F}^{-1}\{\mathcal{F}\{h \cdot r\} \cdot \mathcal{F}\{g\}\}$ \\
\hline $\begin{array}{l}\text { Diffraction integral } \\
\text { (Transfer function) }\end{array}$ & $\mathcal{F}^{-1}\{\mathcal{F}\{h \cdot r\} \cdot G\}$ \\
\hline
\end{tabular}

There is fundamental difference between the Fresnel transform (the evaluation of the given chirp function in the first line of the table) and the given method presented in the following 4 lines. If plane $(\xi, \eta)$,is taken as the spatial field of the digital hologram, then the first process ensures result for spatial frequency domain, because of the sole Fourier transform. The other 4 algorithms comprise the multiplication of spectrum $h \cdot r$ and the transfer function in the spatial frequency domain and from a next transformation.

The result of the distinction is the pixel size diversity in reconstructed images, in compliance with (9) and (16) correlation. Even though of the different sizes, it is still important that size $\Delta x^{\prime} \times \Delta y^{\prime}$ in the Fresnel case uses the chirp function, depending on $\lambda$ wavelength and $d^{\prime}$ reconstruction depth while in the other 4 cases, the size is independent from these parameters. These algorithms are especially well-applicable at inline hologram recordings, where depth information is made from the examined objects. Every reconstructions size allows and provides direct comparison. For size adaptation, there is no need for magnification with final interpolation. These kinds of experiments are described in detail at these processes [10]. 
Theoretically all mentioned methods are attainable in holographic interferometry for giving phase shifts. The two conditions of the examined object which is to be compared are individually recorded and evaluated. The interference phase can be calculated after the evaluation of $b_{1}^{\prime}(n, m)$ and $b_{2}^{\prime}(n, m)$ complex fields phase distribution [1]. There is no significant difference between the 4 methods interference phase distribution, which are based on the convolution approach. If we apply the Fresnel transformation, only the reconstructed image's size will make a contrast from those reconstructed by the convolution approach.

If we examine small objects, theoretically we can work in that field, where the process based on Fresnel approach evoke real/minimal mistakes. Afterwards the convolution approach is suggested, as it gives an exact solution for diffraction integral, until it does not violate the sampling theorem. In the last 4 algorithms of the table, where we take its inverse transform in the spatial frequency field, it brings similar results, however, the numerical process differs. There are such distinctions in the calculation of impulse response or the transfer function fields, where the complex exponential difference differs more than $\pi$ between the neighbouring pixels. In these points the calculated impulse response's or transfer function's absolute value must be set for zero. This method takes place with Fourier transform as well, that outlines the impulse response in the transfer function as well as vice versa. Therefore, the result has nothing to do with the selection of each (above mentioned four) reconstruction algorithm.

\section{CONCLUSION}

The digital hologram recording and numerical reconstruction of holograms provides new opportunities in optical hologram technology. The numerical interpretation of digital Fresnel holograms can be implemented with Fresnel transformation or convolution, in accordance with the diffraction integral. The essential difference between the results of the two approaches resides in the reconstructed image's pixels. If we compare reconstructions with two different depth, the convolution approach is suggested, because here, the image's size does not depend on the depth of the reconstruction. Insofar the examined object is transparent or opaque, the Fresnel transformation is recommended for the reconstruction.

\section{ACKNOWLEDGEMENTS}

The authors would like to thank University Innovation and Research Center, Obuda University, Hungary (EKIK) and the Doctoral School of Applied Informatics and Applied Mathematics, Obuda University, Hungary.

\section{REFERENCES}

[1] Th. Kreis. Holographic Interferometry: Principles and Methods, Akademie-Verlag, Berlin, and VCH Publishers, Inc., New York, 1996.

[2] A. Macovski, S. D. Ramsey, and L. F. Schaefer. Time-lapse interferometry and contouring using television systems. Appi. Opt., 10, 2722-2727, 1971.

[3] J. N. Butters and J. A. Leendertz. Holographic and video techniques applied to engineering measurements. Meas. and Contr., 4, 349-354, 1971.

[4] U. Schnars. Direct phase determination in hologram interferometry with use of digitally recorded holograms. J. Opt. Soc. Amer. A, 11, 2011-2015, 1994.
[5] U. Schnars and W. P. 0. Jptner. Direct recording of holograms by a CCD target and numerical reconstruction. Appi. Opt., 33(2), 179-181, 1994

[6] G. Pedrini, Y. L. Zou, and H. J. Tiziani. Digital double-pulsed holographic interferometry for vibration analysis. J. Mod. Opt., 42(2), 367-374, 1995.

[7] U. Schnars, Th. M. Kreis, and W. P. 0. Jptner. Digital recording and numerical reconstruction of holograms: reduction of the spatial frequency spectrum. Opt. Eng., 35(4), 977-982, 1996.

[8] J. W. Goodman. Introduction to Fourier Optics, McGraw-Hill Companies, Inc., New York, Second Edition 1996.

[9] Th. Kreis, and W. Jptner. The suppression of the d.c.-term in digital holography. To appear in Opt. Eng., 36, 1997.

[10] M. Adams, Th. Kreis, and W. Jflptner. Particle size and position measurement with digital holography. Proc. of European Symposium on Lasers and Optics in Manufacturing, SPIE, 1997. 
Z. Garaguly et al. • Comparison of numerical image reconstruction methods in holography 